\title{
Präsenzcharakter von Krankheit und Spiritual Care
}

Priska Bützberger Zimmerli, Sabine Weidert ${ }^{b}$, Beat Müller ${ }^{c}$

a Dr. med., Oberärztin Onkologie, Kantonsspital Baden

b Dipl. Soz.-Päd., Pflegefachfrau, Koordinatorin Palliativnetz Freiburg i. Br.

c Dr. med., Oberarzt Medizinische Onkologie, Schwerpunktabteilung Palliative Care, Luzerner Kantonsspital
Der Theologe Professor Johannes Fischer schrieb in der SÄZ in einer interessanten Betrachtung zu Krankheit und Spiritualität vom Präsenzcharakter der Krankheit [1]. Was er über die Wahrnehmung der Präsenz der Krankheit schreibt, erinnert an die Ideen der Leibphilosophie von Thomas Fuchs und anderen Leibphilosophen, die den Leib als eigenständiges Wahrnehmungsorgan für Stimmungen, Gefühle und Atmosphären bezeichnen. Er ist darüber hinaus sozusagen der aktuell gespürte Körper. Der Leib wird aber auch als das «Vehikel des ZurWelt-Seins» beschrieben [2]. Eine Krankheit betrifft somit dieses ganze «Vehikel des Zur-Welt-Seins». Folglich verändert sich dadurch die Wahrnehmung der ganzen Welt, wie Fischer es sehr treffend beschrieben hat.

\section{Für Spiritual Care gibt es inzwischen in München einen Lehrstuhl, aber noch keine klare Definition.}

Korrespondenz:

Dr. med. Priska Bützberger

Zimmerli

Kantonsspital Baden

CH-5400 Baden

priska.buetzberger[at]ksb.ch
Er spricht davon, «dass man den Kranken in einen anderen Präsenzraum bringen muss, der Entlastung bietet von dem Präsenz und Erlebensdruck seiner Krankheit». Dies könne der Präsenzraum des Heiligen sein, wie er durch religiöse Praktiken, Riten und Texte erschlossen wird, aber auch einfach der Präsenzraum, die «Atmosphäre» menschlicher $\mathrm{Zu}$ wendung, Fürsorge und Liebe, die einem Kranken entgegengebracht werden [1]. Beides beschreibt etwas, das in der Literatur unter Spiritual Care zusammengefasst wird. In der Leibphilosophie wird dieses Phänomen menschlicher Zuwendung, Fürsorge und Liebe zudem auch als zwischenleibliche Resonanz bezeichnet [3].

\section{Was ist Spiritual Care?}

Für Spiritual Care gibt es inzwischen in München einen Lehrstuhl, aber noch keine klare Definition. Sinngemäss wird damit aber der Aspekt der Gesundheitsfürsorge bezeichnet, der sich um spirituelle und religiöse Bedürfnisse kümmert, die durch eine Krankheit geweckt worden sind. Es gibt Untersuchungen dazu, was sich Patienten unter Spiritual Care vorstellen und was sie sich wünschen [4]. Dabei zeigte sich, dass Spiritual Care für die Patienten sehr viel mit Begegnung und Beziehung zu tun hat, was zu den Erläuterungen von Professor Fischer passt.

Erhard Taverna hingegen schreibt in der Schweizerischen Ärztezeitung: «Reale und vermeintliche Missstände im Medizinbetrieb werden gerne am Ideal der «Begegnung oder des (Paares Arzt-Patient» gemessen, was Michel Foucault als «Bestreben, so vielem Nicht-Denken auch noch die matten Mächte einer Eheträumerei an die Seite zu stellen ironisierte.» Taverna führt weiter aus: «Ganzheitlichkeit oder Spiritualität sind Attribute, die, oft von der alternativen Szene beansprucht, sich hervorragend für die eigene Idealisierung und Selbstbeweihräucherung eignen.» Für Taverna gilt es, der Versuchung, Priester und Heiler sein zu wollen, zu widerstehen [5]. Spiritualität, wie sie z.B. in der Definition der European Association of Palliative Care (EAPC) verstanden wird, hat aber nichts mit Selbstbeweihräucherung zu tun. Die EAPC definiert Spiritualität als «dynamische Dimension menschlichen Lebens, die sich darauf bezieht, wie Personen (individuell und in Gemeinschaft), Sinn, Bedeutung und Transzendenz erfahren, ausdrücken und/oder suchen, und wie sie in Verbindung stehen mit dem Moment, dem eigenen Selbst, mit Anderen/-m, mit der Natur, mit dem Signifikanten und/oder dem Heiligen». [6] Spiritual Care praktizieren $\mathrm{zu}$ wollen, muss nicht gleichbedeutend sein damit, der Versuchung erlegen zu sein, Priester und Heiler gleichzeitig sein zu wollen.

\section{Die Rolle des Helfers}

Der Physiker und Theologe Erhard Weiher schreibt in seinem Buch «Das Geheimnis des Lebens berühren», dass der Helfer sich dem Patienten als Container zur Verfügung stellt. Der Begriff Container stammt dabei aus der Entwicklungspsychologie. So wie das kleine Kind einen Beziehungsraum braucht, um sein Selbst zu finden und auszubilden - ein lebendiges Gegenüber, das ihm tragend und haltend, freilassend und resonanzgebend begegnet - so braucht jeder Mensch immer wieder solche Gegenüber, vor allem in schwierigen Lebensphasen (z. B. Trauer, Sterben). Weiher schreibt über den Patienten: «Wenn er im «Seelenraum» des Helfers auf annehmende Resonanz stösst und sich verstanden und wertgeschätzt findet mit allem, was in ihm ist, dann entsteht für ihn ein verlässlicher Pol, an dem er 


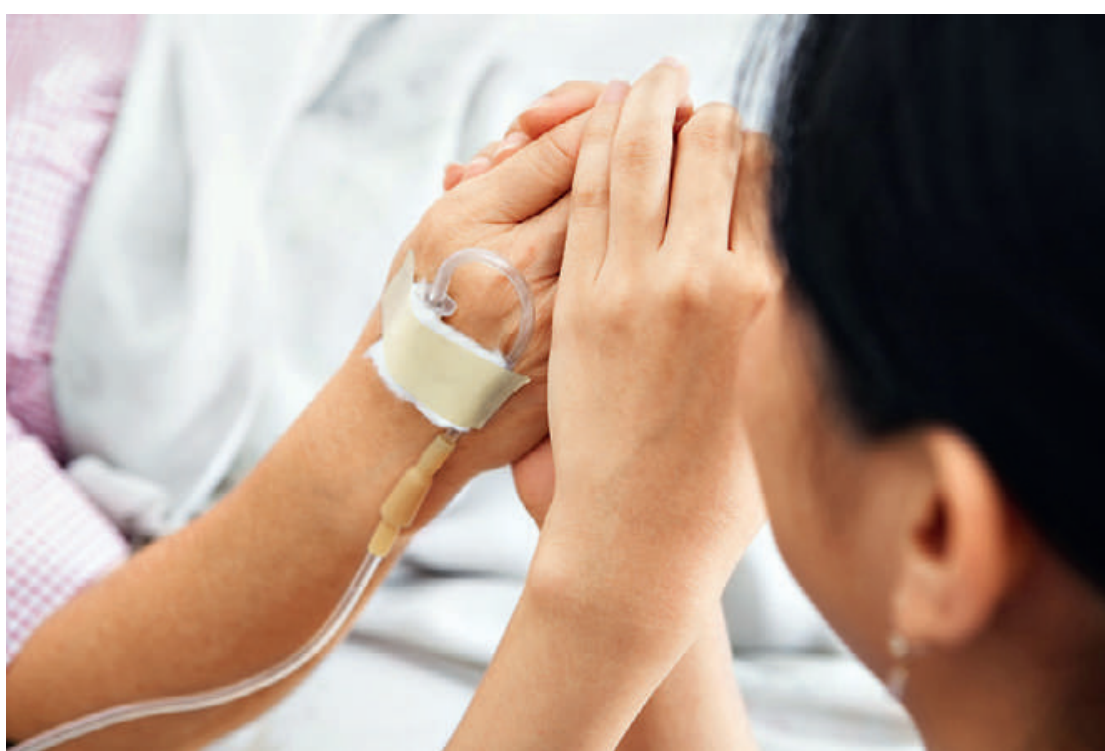

Beim Helfer erhält der Patient Resonanz, fühlt sich verstanden und wertgeschätzt, findet einen verlässlichen Pol, an dem er sich aufrichten und wieder zu sich finden kann.

sich aufrichten und wieder zu sich finden kann.» [7] Taverna befürchtet eine Überforderung der Behandelnden und Pflegenden. Weiher schreibt dazu: «Die Begleiter sind für den Patienten nicht nur Auffanggefässe (...). Ihnen steht selbst ein Container zur Verfügung, aus dem sie ihrerseits die beruflichen Ressourcen schöpfen können, um schwerer Krankheit, Sterben und Tod zu begegnen. Der Container des Helfers ist nicht voller hilfloser Ohnmacht (und damit leer), sondern weit über das fachlich-funktionelle Denken und Handeln hinaus gefüllt mit Inhalten, die das Leid von Menschen tragen helfen, so dass die Helfer das Wesentliche nicht mit eigener Kraft tragen müssen.»

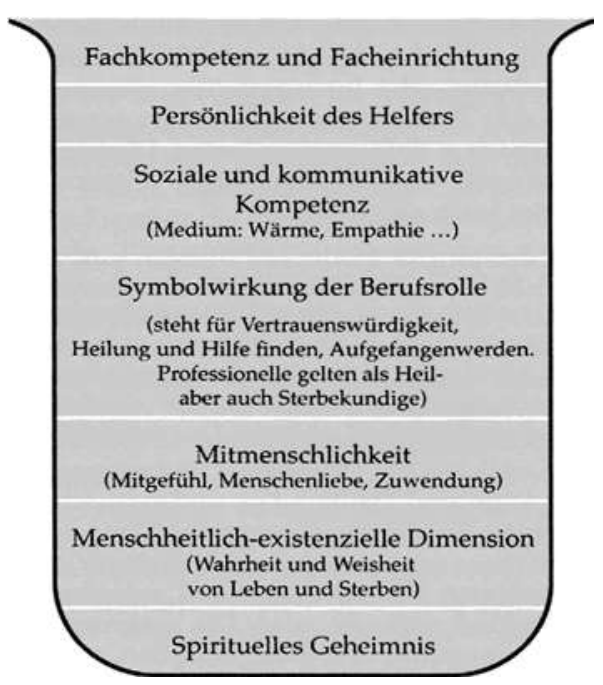

Der Container und seine Auffangstruktur. (Aus: Erhard Weiher. Das Geheimnis des Lebens berühren. Mit freundlicher Genehmigung des Kohlhammer-Verlags.)
Die oberste Ebene dieses Containers ist gemäss Weiher mit dem fachlich-methodischen Handeln und Behandeln gefüllt. Der Helfer, z.B. der Arzt, wendet sich mit seiner Fachkompetenz der physischen Seite des Patienten zu. Dies geschieht im Rahmen einer Facheinrichtung, die wiederum Teil der Gesellschaft ist. Die zweite Ebene stellt die Persönlichkeit des Helfers dar. Sie verleiht der beruflichen Rolle Farbe und ein persönliches Gesicht. Bei der dritten Ebene wird die Beziehungskompetenz angesprochen. In der Leibphilosophie käme hier die leibliche Resonanzfähigkeit mit ins Spiel, d.h. die Schwingungsfähigkeit des Lei- bes für die Wahrnehmung von Atmosphären, Stimmungen und Gefühlen [8]. Bei der vierten Ebene geht es um die Symbolwirkung der Berufsrolle. Der Arzt z. B. ist ein Vertreter der Gesellschaft, dem diese bedeutungsvolle Berufsrolle zugetraut wird und der auch dafür steht, dass man mit seinem Leiden aufgefangen wird. Folgt die Ebene der Mitmenschlichkeit. Ein Mensch begegnet einem anderen Menschen. Im Container gibt es auch eine Schicht menschheitlichexistentieller Grunderfahrung. «Die Fähigkeit, mit Leid und Tod zu leben, gehört von Anfang der Schöpfung an zur Natur der Lebewesen.» Die unterste Schicht reicht in die spirituelle Dimension hinein. «Leben, Leiden, Sterben und Tod sind in dem Geheimnis allen Lebens verankert.» Der Mensch verfügt nicht über dieses Geheimnis des Lebens, aber er kann sich ihm anvertrauen und es bewohnen [7].

Taverna schreibt, die Begriffe seien unscharf. Es stellt sich die Frage, ob Begriffe wie Spiritualität oder Spiritual Care überhaupt scharf umrissen sein können, haben sie doch mit dem «spirituellen Geheimnis» des Lebens zu tun, das für uns Menschen unverfügbar ist.

\section{Fazit}

Zusammenfassend gibt es durchaus einen Mittelweg zwischen der Reduktion der Krankheit auf somatische oder psychische Zustände und dem Abdriften in Selbstbeweihräucherung und Heilerfantasien. Eine ärztliche Spiritual Care mit der nötigen Demut, wie sie von Weiher beschrieben wird und ausgehend vom Gedanken, dass jeder Mensch in irgendeiner Form spirituell ist, kann durchaus auch präventiv wirken gegen ein solches Abdriften in Allmachtsund Grössenfantasien.

\section{Literatur}

1 Fischer J. Krankheit und Spiritualität. Schweiz Ärztezeitung. 2012;93(45):1672-5.

2 Merleau-Ponty M. Phänomenologie der Wahrnehmung. Berlin; 1965. S. 106.

3 Fuchs T. Leib, Raum, Person, Entwurf einer phänomenologischen Anthropologie. Stuttgart; 2000. S. 246.

4 Edwards A et al. The understanding of spirituality and the potential role of spiritual care in end-of-life and palliative care: a meta-study of qualitative research. Palliat Med. 2010;24(8):753-70

5 Taverna E. Mehrwert Spiritualität. Schweiz Ärztezeitung. 2012;93(45):1678.

6 European Association of Palliative Care (Internet). (The Netherlands): Taskforce on Spiritual Care in Palliative Care; (2010, cited 2012 Nov 14). www. eapcnet.eu

7 Weiher E. Das Geheimnis des Lebens berühren. Stuttgart: Kohlhammer; 2009.

8 Weidert S. Leiblichkeit in der Pflege von Menschen mit Demenz. Frankfurt a. M.: Mabuse-Verlag; 2007. 